\title{
Mdct Assessment of Major Aorto-Pulmonarycollaterals
}

\author{
Dr.Subhashree Chandrasekan ${ }^{1}$, Dr. Kannadhasan Ramadhas², Dr.Kanaga Durga \\ $S^{3}$ \\ ${ }^{1,2,3}$ (Department Of Radiodiagnosis, Coimbatore Medical College, Tamilnadu, India)
}

\begin{abstract}
Objective: To describe the importance of performing MDCT(Multidetector-row computed tomography) in the management of patients with pulmonary atresia-ventricular septal defect(PA-VSD) and to assess the variations in pulmonary arterial circulation, assessment of major aorto-pulmonary collaterals and other associated anomalies of the cardiovascular system.

Methodology: MDCT with intravenous contrast was performed in three adult patients, two of them presented with cyanotic spells and one patient presented with dyspnoea. Various image reformatting techniques including maximum intensity projection (MIP) and curved multi-planar reconstruction(MPR) and volume-rendering technique (VRT) were used.

Results: In these three patients, the main pulmonary arteries were congenitally absent, and the blood supply to pulmonary circulation was totally carried out by major aorto-pulmonary collateral arteries (MAPCAs). MDCT provided detailed road map for evaluation of MAPCAs, estimation of the number and site of origin, their diameters, courses, and the lobes of the lungs supplied by them. MDCT could provide an efficient road map to guide safe selective catheterization and could be a substitute for conventional angiography.

Conclusion: The management plan of this particular congenital cardiac malformation is mainly determined by the morphology of main pulmonary arteries and the presence of extra-cardiac sources of pulmonary blood supply and the vasculature of the lung parenchyma is well assessed with MDCT which is the non-invasive imaging technique of choice in the detailed evaluation of this condition.
\end{abstract}

\section{Introduction}

Pulmonary atresia with ventricular septal defect refers to a group of congenital cardiac anomalies, in which there is lack of continuity between the pulmonary arteries and ventricle, due to pulmonary atresia with an associated ventricular septal defect. There can be atresia of the entire central pulmonary artery or a part of it ${ }^{[1]}$. The lung is supplied by the collateral vessels that originate from extracardiac sources, which could originate from a patent ductus arteriosus (PDA) or from major aorto-pulmonary collateral arteries (MAPCAs), which connect the systemic circulation to the pulmonary arteries. This congenital malformation is also referred as "pseudo-truncus arteriosus," and "truncus arteriosus type 4". A subtype of Tetralogy of Fallot (TOF) can be associated with marked infundibular stenosis, in which there may be complete obstruction of pulmonary blood flow $^{[2]}$. There is total separation of the right ventricle from the pulmonary arteries, which requires the definite presence of extra-cardiac sources of pulmonary arterial blood flow to maintain the viability of the individual.

On the basis of the characteristics of the pulmonary circulation, PA-VSD is classified into three types ${ }^{[3]}$. Type A, the native pulmonary arteries are present which are supplied by PDA. Type B, pulmonary blood flow is provided by both native pulmonary arteries and by MAPCAs. In type C, native pulmonary arteries are absent and the blood supply is only through MAPCAs. All the three cases in our observation belonged to the type $\mathrm{C}$ group.

Appropriate assessment of this condition is essential as the surgical treatment of this condition is determined by the morphology of pulmonary arteries. The presence of large native pulmonary arteries and no MAPCAs permits the performance of palliative procedures, such as the performance of aortopulmonary shunt in younger children and corrective surgery with right-ventricle-to-pulmonary-artery continuity in older children. Patients with short-segment pulmonary atresia can undergo intracardiac repair without conduit. But, if there are multiple MAPCAs, single or multi-stage rehabilitation of pulmonary arteries in which connecting all MAPCAs to native pulmonary arteries is needed before intracardiac repair ${ }^{[4,5]}$. Appropriate characterization of thestatus of pulmonary arteries and MAPCAs is of paramount importance in management of the patients with PA-VSD ${ }^{[6]}$. Echocardiography could precisely demarcate the anatomy of the central pulmonary arteries in infants with PAVSD, but echocardiography has its own limitations in older children and adults because of poor acoustic windows and echocardiography could not provide the surgeon with the precise anatomic road mapping of MAPACAs.

With the introduction of MDCT the preoperative imaging of patients with PA-VSD has become easier and precise. It provides a detailed and non-invasive imaging of the pulmonary arteries, the aorta, the cardiac 
chambers and the collateral vessels. Through 3D imaging software a complex pulmonary blood supply can be noninvasively and accurately imaged with $\operatorname{MDCT}^{[7]}$.

\section{Imaging Techniques}

Short-term IV sedation is often required, particularly in children under 5 years of age, but in our study all the three patients were adults in the third decade and hence sedation was not required. All the procedures were performed with MDCT scanner (TOSHIBA). The radiation dose was kept to the minimum by reducing the kilovoltage and tube current appropriately. Contrast media was injected to achieve homogeneous vascular enhancement along with image acquisition. To achieve greater image quality adequate care was taken regarding the IV access, dose and density of contrast material and rate of injection. Wherever possible, a leg vein is preferred to avoid streak artefacts from high-density contrast material in the upper limb veins. Appropriate cannula size should be selected to achieve a high rate of contrast injection. Iohexol 350 (Omnipaque) at a dose of $1.5 \mathrm{~mL} / \mathrm{kg}$ was used with an injection rate of $1.5-2.0 \mathrm{~mL} / \mathrm{sec}$.Using the bolus-tracking technique, the scanning can be started manually after seeing the contrast medium in the right ventricle. A minimum delay of 4 seconds is used to start the scan after the contrast medium reaches the right ventricle. Because of the presence of ventricular septal defect (VSD) homogeneous contrast enhancement of both pulmonary and systemic arteries can be achieved using this method. The CT can be repeated 5-8 seconds after the first phase if there is suspicion about systemic venous anomalies.

CT is performed from the thoracic inlet level to the level of L1-L2. ECG gating is not used routinely for younger children because it increases radiation dose and increases scan time, which produces more respiratory artifacts. But, most of the extracardiac anatomy can be adequately depicted without ECG gating. Even though distal coronary arteries cannot be visualized without ECG gating, nongated MDCT images are sufficient to identify the origin and to evaluate the proximal course of the major arteries, which is sufficient for management of these patients. All image data are evaluated using 3D post-processing workstation (Osirix) with various image reformatting techniques including curved planar reconstruction, maximum intensity projection (MIP), and volume-rendering technique (VRT) are used to get all the necessary clinically relevant information.

\section{Mdet Evaluation Of Pa-Vsd}

The imaging assessment of PA-VSD includes assessment of the pulmonary arteries, the other sources of pulmonary blood supply, MAPCAs, aortic arch anomalies, situs and the chambers of heart. Analysis of the pulmonary artery anatomy is the single most important aspect of diagnostic imaging of patients with PA-VSD. Several studies have shown that MDCT is the ideal and accurate tool for evaluating pulmonary arteries. The length of the pulmonary atresia, the pulmonary artery confluence, the size of main, right, and left pulmonary arteries at the origin and at the hilum are recorded. Images were also carefully analyzed for branch pulmonary artery stenosis. The sources of pulmonary blood flow to each lung, number of bronchopulmonary segments supplied by native pulmonary arteries, and the distribution of each MAPCA must be assessed to guide the surgical treatment. It is of prime importance to map the source of supply to each part of the lung using the MDCT data. In all our three cases the main pulmonary arteries were absent and the lungs were predominantly supplied by the collaterals. The assessment of PA-VSD includes assessment of the situs, pulmonary arteries, aortic arch, ventricular septal defect and assessment of MAPCAs ${ }^{[8]}$.

Situs anomalies may be seen in association with PA-VSD, hence they may be associated with asplenia, central liver, right-sided stomach, or left-sided IVC. All our three cases had a normal situs.

The other associated congenital anomalies of the heart could be detected and in our case the over-riding of aorta and subaortic VSD were first seen in MDCT.

MDCT provides an accurate delineation of the presence of various associated aortic anomalies, which include a right-sided aortic arch, aberrant right or left subclavian artery, coarctation of the aorta, and double aortic arch is essential as these variations are important to determine the surgical approach for a shunt.

MAPCAs represent fetal primitive intersegmental arteries that originate from the descending aorta. Normally during fetal development when the pulmonary vasculature forms well, these arteries begin to involute. When there is defect in this pulmonary vasculature development, these MAPCAs persist. They gain access to the lung through the hilum and connect with native pulmonary arteries in the mediastinum or at the lobar or subsegmental level. By using various MDCT postprocessing techniques, the total number of MAPCAs and their origin, course, and intrapulmonary connections could be easily assessed.

Pleural pulmonary collaterals are the other systemic branches which are seen supplying the thoracic wall can perfuse the intrapulmonary arteries across the pleural connections. Another source of pulmonary blood supply include the mediastinal collaterals originating from the subclavian arteries and coronary-pulmonary collaterals from branches of coronary arteries through atrial, pericardial, and mediastinal branches

Knowledge of the adequacy of the pulmonary blood supply to each bronchopulmonary segment is essential before doing major corrective surgery. When both MAPCAs and native pulmonary arteries supply a 
bronchopulmonary segment, ligations of MAPCAs do not affect lung arborization. If a bronchopulmonary segment is exclusively supplied by MAPCAs, then that segment needs to be unifocalized in order to preserve the blood supply to the broncho-pulmonary segment ${ }^{[9,10]}$. Communication between MAPCAs and native pulmonary arteries can occur with central pulmonary arteries, at the hilum or major lobar branches, and at segmental or subsegmental levels.

Coronary anomalies associated with PA-VSD are aberrant origin of the left anterior descending artery from the right coronary artery coursing anterior to the infundibulum of the right ventricle, high origin of the coronary ostia, coronary-artery-to-pulmonary-artery fistula, and aberrant right coronary origin from the left aortic sinus.

\section{Case Report}

A 32 year old male presented with complaints of dyspnoea and episodes of cyanotic spells for past three years. On examination, the patient had grade three dyspnoea, central cyanosis and clubbing. The patient underwent imaging through chest radiograph, echocardiography, and MDCT angiography of chest. Systemic examination revealed systolic murmur in precordium. Chest x-ray showed the presence of cardiomegaly, pulmonary plethora and absent pulmonary conus. Echo showed the presence of dilatation of all the cardiac chambers, overriding of aorta and a large non restrictive VSD. MDCT evaluation of the patient revealed dilated cardiac chambers, overriding of aorta, subaortic VSD measuring $2.5 \mathrm{~cm}$ in size, infundibular stenosis and absent main pulmonary artery. The right and left pulmonary arteries were reformed by the multiple MAPCAs. These MAPCAs were seen originating from the thoracic and suprarenal abdominal aorta, coronary arteries and mediastinal and pleural collaterals. There were multiple collaterals arising from the ascending, arch, descending and suprarenal aorta that supplied both lobes of lung. Due to the development of complicated intrathoracic collateral circulation, patient was referred to the higher centre for further management.

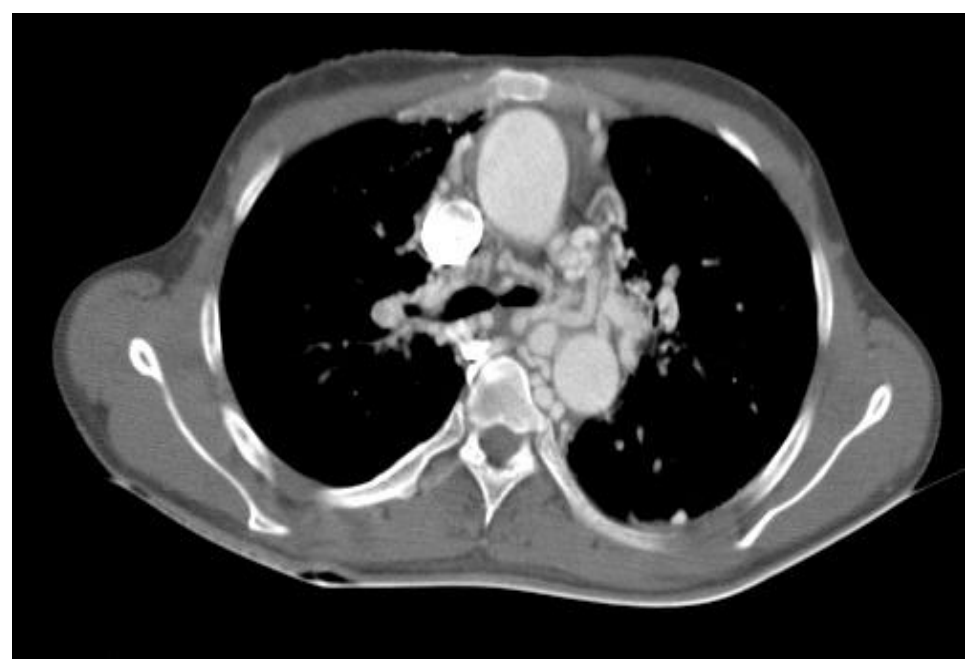

Figure1. Absence of main pulmonary artery

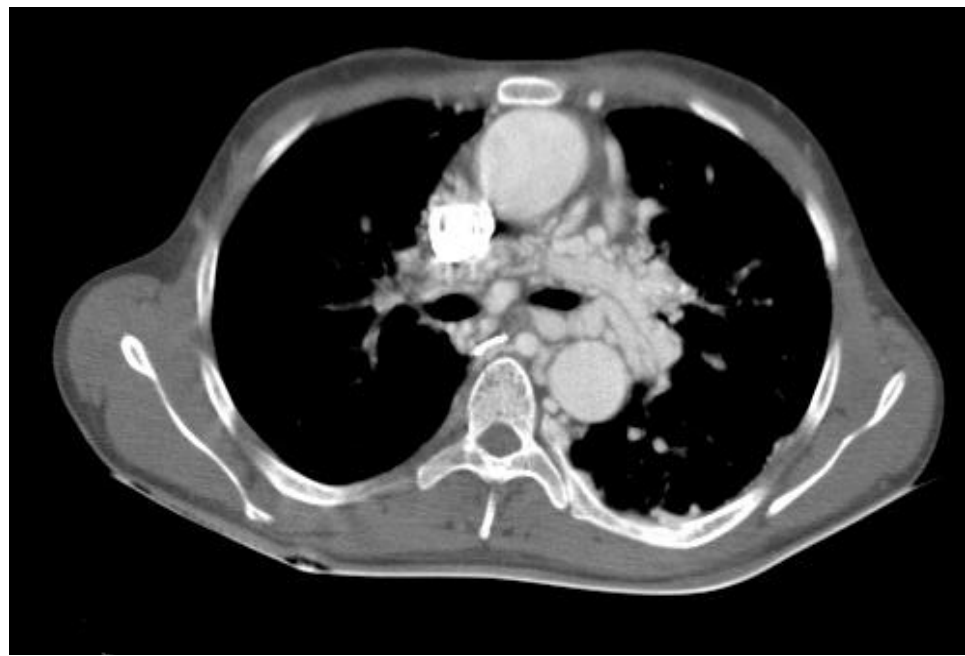

Figure 2. Right and left pulmonary arteries are reformed through MAPCAs 


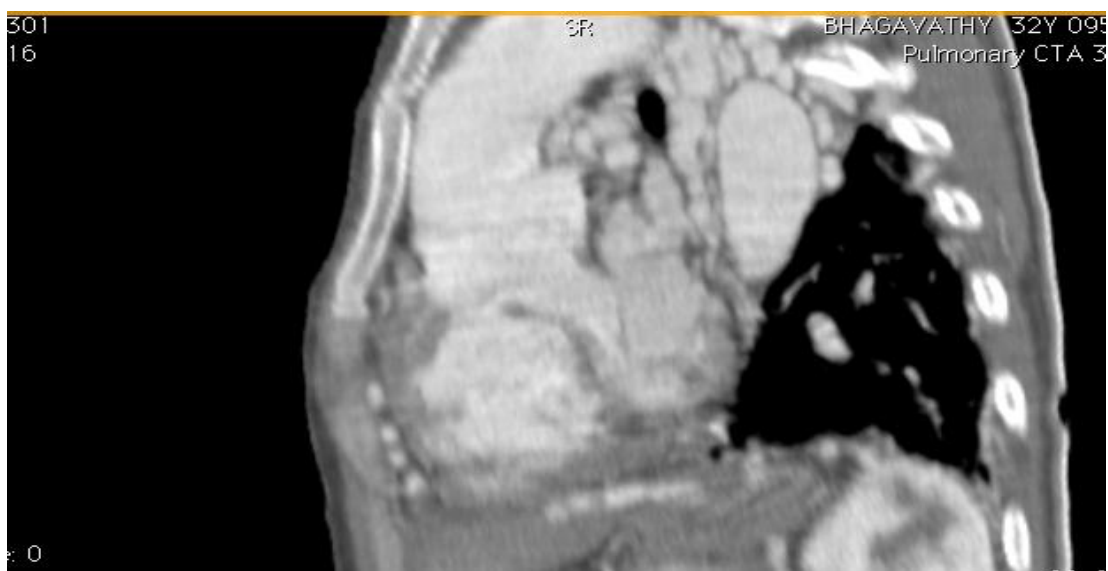

Figure 3.over-riding of aorta

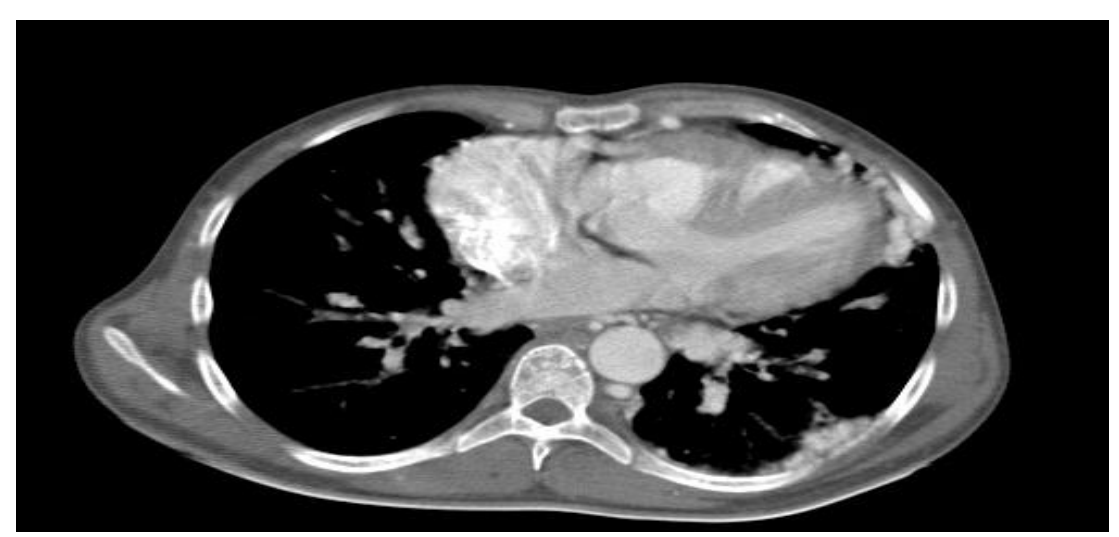

Figure 5.subaortic VSD

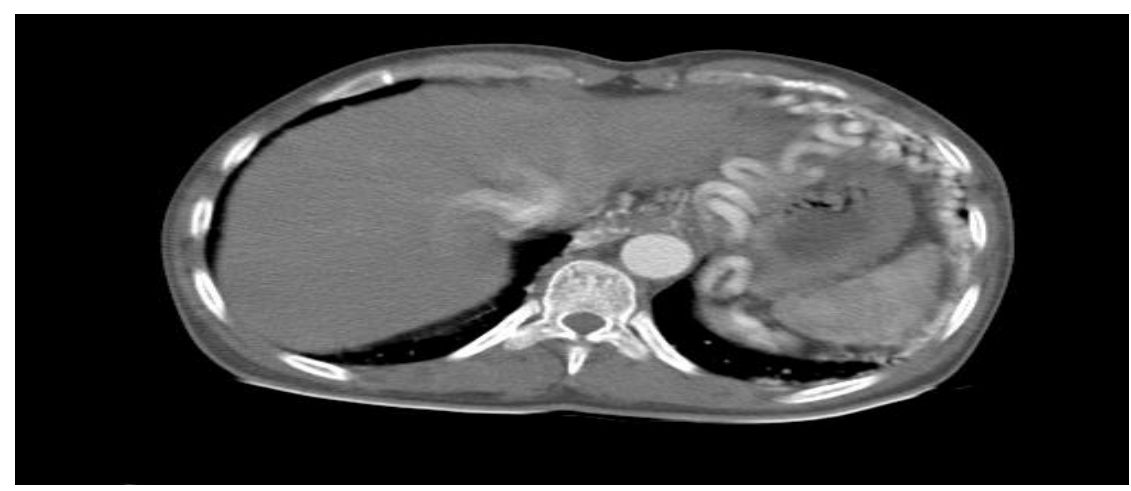

Figure 6. MAPCAs arising from supra-renal abdominal aorta

The second case, a 21-year-old female presented with a history of severe breathlessness in past one year. There was no previous history of similar complaints during the rest of her pregnancy, her childhood, and adolescence. Clinical examination revealed grade I clubbing but there was no evidence of cyanosis. Systemic examination revealed systolic murmur in the precordium. Chest X-ray revealed left hilar prominence, right sided aortic arch and absent pulmonary conus. Transthoracic 2D echo revealed a single large nonrestrictive ventricular septal defect with right-to-left shunt, overriding of aorta and pulmonary atresia. CT pulmonary angiography revealed absent main pulmonary artery, right pulmonary artery, and left pulmonary artery with non-visualisation of the right ventricular outflow tract indicating pulmonary atresia. Large arterial channels representing MAPCAs (2 on right and 1 on left) were seen arising from the aorta (one arterial channel arising from the descending thoracic aorta supplying blood to the RUL, one arterial channel seen arising from the arch of aorta supplying blood to the RML and RLL, and another large tortuous arterial channel arising from the descending thoracic aorta supplying the LUL and LLL). There was large anterior 2.4-centimetre sized subaortic VSD, overriding of aorta, and right ventricular hypertrophy confirming the echocardiography findings. The absence of the native pulmonary vascular channels with MAPCAs supplying the lung directly indicates a type C PA-VSD. There was presence of right sided aortic arch with dilated ascending aorta, arch of aorta and descending thoracic 
aorta. Since the patient had a significant chance of developing pulmonary obstructive vascular disease later, so a multistep surgical approach was planned for this patient which comprises unifocalization, that is, unification of the MAPCAs followed by establishment of right ventricle to pulmonary artery continuity and closure of the VSD.

The third case was a21-year-old male patient with chief complaint of breathlessness and discoloration of fingers. In the past there was a gradual increase in difficulty in breathing with exertional dyspnea and palpitation. No surgical history in the past. The patient underwent for imaging study including chest radiograph, echocardiography, and multidetector computed tomography (MDCT) angiography of the chest. Chest radiograph of patient revealed characteristic "boot shaped heart" sign. No significant pulmonary oligemia was seen. Echocardiography study revealed large ventricular septal defect with poor detection of pulmonary artery. Three-dimensional MDCT and angiography of chest performed using bolus tracking technique followed by multiplanar reconstruction. The CT study demonstrated the absence of main pulmonary trunk with small hypoplastic native pulmonary arteries, large patent ductus arteriosus (PDA), ventricular septal defect VSD, overriding of the aorta with aortic root dilatation and RVH. Furthermore, major aortopulmonary collateral arteries (MAPCA) arising from the descending aorta, right subclavian artery, large PDA feeding the native pulmonary arteries and a dilatation of the aortic root were observed. Vascular mapping performed with the help of MPR and 3D CT technique. A large PDA was seen forming a collateral arterial arch with the left-sided native pulmonary artery and responsible for the blood supply in native intercommunicating pulmonary arteries. Due to the presence of complicated intrathoracic collateral circulation, patient was referred to the higher center for further management.

\section{Limitations Of Mdet}

The main disadvantage of MDCT is radiation exposure, a factor to be considered particularly in regard to children and the radiation exposure as low as possible during scanning. We found that all clinically relevant diagnostic information can be obtained using low-dose non-ECG-gated MDCT.A drawback of non-ECG-gated MDCT compared with catheter angiography is that it is a relatively static imaging method best suited for morphologic assessment. For functional assessment, such as ejection fraction and regional wall motion, ECGgated images are required, which will considerably increase the radiation dose tothe patient.

\section{Conclusion}

MDCT proved as a valuable tool in comprehensive evaluation of different anatomic structures, including the heart, pulmonary and systemic thoracic vasculature, lungs, and abdomen, when evaluating patients with PA-VSD. Multiplanar and 3D CT reconstruction images can improve communication of anatomic details to clinicians. MDCT has proved to be an invaluable diagnostic and decision-making tool as a compliment to echocardiography and increasingly as a substitute for invasive angiography in the management of PA-VSD.

\section{References}

[1]. R. Rajeshkannan, S. Moorthy, K. P. Sreekumar, P. V. Ramachandran, R. K. Kumar, and K. S. Remadevi, "Role of 64-MDCT in evaluation of pulmonary atresia with ventricular septal defect," American Journal of Roentgenology, vol. 194, no. 1, pp. 110-118, 2010 .

[2]. C. I. Tchervenkov and N. Roy, "Congenital heart surgery nomenclature and database project: pulmonary atresia-ventricular septal defect," The Annals of Thoracic Surgery, vol. 69, supplement 1, no. 3, pp. S97-S105, 2000

[3]. Liao PK, Edwards WD, Julsrud PR, Puga FJ, Danielson GK, Feldt RH. Pulmonary blood supply in patients with pulmonary atresia and ventricular septal defect.J Am CollCardiol 1985; 6:1343-1350

[4]. Ishibashi N, Shin'oka T, Ishiyama M, Sakamoto T, Kurosawa H. Clinical results of staged repair with complete unifocalization for pulmonary atresia with ventricular septal defect and major aortopulmonary collateral arteries. Eur J CardiothoracSurg 2007; 32:202-208

[5]. Duncan BW, Mee RB, Prieto LR, et al. Staged repair of tetralogy of Fallot with pulmonary atresia and major aortopulmonary collateral arteries. J ThoracCardiovascSurg 2003; 126:694-70

[6]. Gupta A, Odim J, Levi D, Chang RK, Laks H. Staged repair of pulmonary atresia with ventricular septal defect and major aortopulmonary collateral arteries: experience with 104 patients. J ThoracCardiovascSurg 2003; 126:1746 -175

[7]. Paul JF, Lambert V, Losay J, et al. Three-dimensional multislice CT scanner: value in patients with pulmonary atresia with septal defect [in French]. Arch Mal Coeur Vaiss 2002; 95:427-432

[8]. P.-K. Liao, W. D. Edwards, P. R. Julsrud, F. J. Puga, G. K. Danielson, and R. H. Feldt, "Pulmonary blood supply in patients with pulmonary atresia and ventricular septal defect," Journal of the American College of Cardiology, vol. 6, no. 6, pp. 1343-1350, 1985

[9]. N. Ishibashi, T. Shin'oka, M. Ishiyama, T. Sakamoto, and H. Kurosawa, "Clinical results of staged repair with complete unifocalization for pulmonary atresia with ventricular septal defect and major aortopulmonary collateral arteries," European Journal of Cardio-Thoracic Surgery, vol. 32, no. 2, pp. 202-208, 2007.

[10]. Devendra V. Kulkarni, Rahul G. Hegde, AnkitBalani, and Anagha R. Joshi "A Rare Case of Pulmonary Atresia with Ventricular Septal Defect with a Right Sided Aortic Arch and a Calcified Pulmonary AVM Presenting in an Adult without Cyanosis" Hindawi Journal of Radiology-2014 